\title{
Éducation à l'environnement et institution scolaire
}

Jean-Étienne Bidou

\section{(2) OpenEdition}

Journals

Édition électronique

URL : http://journals.openedition.org/ere/3888

DOI : $10.4000 /$ ere.3888

ISSN : 2561-2271

Éditeur

Centr'ERE

Référence électronique

Jean-Étienne Bidou, «Éducation à l'environnement et institution scolaire », Éducation relative à

l'environnement [En ligne], Volume 6 | 2007, mis en ligne le 14 septembre 2007, consulté le 24 septembre 2020. URL : http://journals.openedition.org/ere/3888 ; DOI : https://doi.org/10.4000/ere. 3888

Ce document a été généré automatiquement le 24 septembre 2020. 


\title{
Éducation à l'environnement et institution scolaire
}

\author{
Jean-Étienne Bidou
}

1 Nous vivons une situation paradoxale. Alors que l'éducation à l'environnement est progressivement prise en compte dans la plupart des systèmes scolaires francophones, les inquiétudes se manifestent quant à la forme que prend cette intégration. En France, par exemple, l'éducation à l'environnement, après une phase de tolérance et même d'encouragement sous la forme de projets scolaires, est entrée récemment de façon très officielle dans les cursus; mais elle l'a fait sous la forme de l'EEDD (éducation à l'environnement pour un développement durable), qui doit beaucoup à l'intérêt actuel pour le développement durable. Dès lors, chez les praticiens, les esprits sont fort partagés, entre la satisfaction liée à la reconnaissance de l'éducation à l'environnement désormais par l'institution et la crainte de voir ses aspects essentiels fortement altérés. Ainsi ressurgit le problème des rapports difficiles entre un mouvement éducatif et l'institution.

2 L'école est en crise ; au moins le dit-on beaucoup. Il est vrai qu'elle a beaucoup changé et qu'elle a accompli au cours des dernières trois ou quatre décennies une mutation spectaculaire après des choix politiques majeurs qui en ont fait une école de masse. Crise, mutation forcée des méthodes, des structures, et probablement des finalités même de l'école ; le fait est que la nécessité de s'adapter à des problématiques nouvelles a conduit à placer l'innovation au cœur du système. Qu'elle provienne d'initiatives du corps enseignant ou qu'elle fasse partie d'une réforme, l'innovation est désormais instituée comme politique ou mode de gestion au moins dans le discours sinon dans les faits. Et à ce titre, l'introduction de l'éducation à l'environnement peut d'abord être considérée comme une innovation.

3 En effet, l'éducation à l'environnement, malgré son ancienneté relative, peut encore être considérée comme une nouveauté. Comme toute innovation qui vise à combler un manque ou améliorer une situation jugée insatisfaisante, l'éducation à l'environnement a été, à ses débuts, un effort pour amener à une prise de conscience des problèmes environnementaux qui se posaient localement ou globalement. Elle l'a fait en traçant 
une démarche qui remettait en cause une pédagogie considérée comme traditionnelle. Pour impulser une dynamique de changement, elle demandait à ses acteurs un engagement personnel. Enfin, comme toute innovation, l'éducation à l'environnement s'est placée d'abord à la marge de l'institution, souvent sous la forme de dispositifs expérimentaux, plus tard officialisés ou tombés en déshérence. Ces praticiens de l'éducation à l'environnement ont été décrits comme des innovateurs dans le système scolaire : ils partagent leur implication, leur goût et leur croyance assez générale dans l'efficacité du travail d'équipe, et l'intérêt dans la pratique de la réflexivité.

ais les innovateurs, ainsi que le rappelle F. Cros (2004), changent les règles du jeu alors qu'ils ne sont pas en position institutionnelle pour le faire. D'où ce jeu quelquefois un peu pervers entre la séduction des innovateurs qui peuvent se laisser aller à des compromis pour la reconnaissance de leurs idées et les hésitations des représentants de l'administration tentés par un changement bien nécessaire sous peine de sclérose et la crainte d'aller trop loin. En effet, certaines innovations sont acceptables, et d'ailleurs sont rapidement intégrées. D'autres, "plus militantes, dont le combat porte sur la proposition d'une société résolument alternative, auront du mal à s'inscrire dans ce schéma » (Cros, 2004). C'est donc dans ce cadre que peut se poser la question du statut de l'éducation à l'environnement et de ses difficultés à pénétrer l'institution scolaire : trente ans d'efforts pour finalement entrer dans le cursus français au titre du développement durable. Qu'est-ce donc qui rendrait l'éducation au développement durable plus aisément assimilable que l'éducation à l'environnement?

5 L'éducation à l'environnement se distingue d'une éducation où la perspective du développement durable serait introduite par au moins deux points essentiels.

Le premier est d'ordre éthique. L'éthique du développement durable est strictement anthropocentrée. La place centrale qu'occupent les humains dans le monde leur confère la responsabilité de soi, d'autrui et du monde. Si la plupart des praticiens de l'éducation à l'environnement partagent aussi cette vision, elle n'est pas la seule. Le biocentrisme, qui accorde à la Nature une valeur propre y est également valide; il procède de la conscience de l'interdépendance qui rassemble et conditionne l'existence de tous les êtres vivants, l'Homme compris.

7 Le second est lié à la posture politique. L'éducation à l'environnement permet aussi une assez grande diversité d'options, même si actuellement dominent celles qui ressortent du paradigme sociocritique. Or, de ce point de vue, on ne peut manquer de remarquer le synchronisme entre la diffusion du développement durable et le triomphe de l'économie libérale dans un monde globalisé. Alors que dans la perspective du développement durable, l'accent est mis sur les résiliences, les régulations, les recherches de compromis entre acteurs, l'éducation à l'environnement, même si dans les faits elle ne l'exerce guère, garde la liberté d'une critique radicale d'un système économique (ou social, d'ailleurs) et de la confrontation.

8 La politique, aujourd'hui comme autrefois est l'art du possible et les réformes éducatives doivent tenir compte du contexte dans lequel elles s'appliquent, des résistances et des moyens qu'elles peuvent mobiliser. Le contexte est celui d'une école en proie à une crise aux dimensions multiples : celle des méthodes, de l'autorité, des contenus et finalement même du sens. Les résistances aux transformations sont multiples, et sans stigmatiser enseignants, administrateurs, parents d'élèves et autres groupes, le débat sur l'école voit s'opposer partout une logique de reproduction à l'identique et l'injonction à la réforme ininterrompue, ce qu'on a pu qualifier en France 
de querelle des "républicains » et des "pédagos». Dans ces conditions, les marges de manœuvre sont relativement étroites, et à moins d'une réforme radicale qui aurait peu de chance d'être adoptée par toutes les parties prenantes, l'institution est bien contrainte de choisir les lignes de moindre résistance. Celles-ci sont à peu près les mêmes dans les différents systèmes nationaux.

9 L'aspect matériel est probablement le plus consensuel: mise aux normes environnementales et gestion plus écologique des établissements, hélas rarement dans une perspective holiste comme le souhaite un des contributeurs de l'ouvrage.

À l'heure actuelle, l'ancrage de l'éducation à l'environnement se fait en général à l'intérieur des programmes disciplinaires. On peut, et c'est le plus simple, utiliser le développement durable dans des thèmes disciplinaires, par exemple en biologie, physique ou en géographie. Mais les savoirs liés à l'environnement sont par définition transdisciplinaires, complexes et portent une incertitude dont il faut tenir compte dans les démarches, qu'elles soient scientifiques ou éducatives. La pratique de l'interdisciplinarité demeure problématique, même à partir de disciplines voisines. En outre l'éducation à l'environnement introduit la complexité et l'incertitude. L'une et l'autre questionnent l'institution scolaire: comment aborder des savoirs incertains dans le cadre d'une classe? Quel statut donner à l'erreur et à l'ignorance ?

11 La solution pédagogique de ces problèmes impose le plus souvent le recours à des apprentissages de type socioconstructiviste auxquels peu d'enseignants sont actuellement formés.

12 La formation des éducateurs devient donc un problème crucial. La majorité des enseignants ne pratique pas l'éducation à l'environnement, et certains la subissent plutôt : plusieurs contributions ont montré le désarroi des enseignants qui voient se désagréger une partie de leurs certitudes quant à leur enseignement et à leurs pratiques. Ainsi, l'introduction de l'éducation à l'environnement accompagne le malaise et la remise en question de la position des enseignants et la fonction de l'école. Cela ne rend pas sa généralisation plus aisée.

13 L'introduction de l'éducation à l'environnement entraîne souvent l'ouverture des structures éducatives: la classe à l'occasion d'un projet, l'établissement pour une opération de type Agenda 21 ou Éco-école par exemple. L'école s'ouvre à des partenaires locaux ou plus lointains: institutions, collectivités, associations, entreprises. La nature et le fonctionnement de ces partenariats se révèlent parfois discutables. Enfin, dans la hiérarchie scolaire se rencontrent des résistances dont les plus farouches ne sont pas forcément les mieux avouées.

14 Finalement, au-delà de la très grande variété des expériences, les contributions de cet ouvrage rendent compte des progrès mesurés de l'éducation à l'environnement à l'intérieur de l'institution, de la percolation lente des thèmes et des méthodes, des difficultés de leur mise en place, des enjeux des réformes. Ce tableau est celui de la résistance d'une institution en plein doute sur ses fonctions. Mais faut-il rappeler que les institutions sont des formes d'organisation sociale, construites par les individus pour pouvoir vivre ensemble ? L'institution c'est aussi nous qui la construisons. Dès lors il nous faut être capables de dialoguer avec elle, de rappeler nos positions, de proposer des réformes, d'incarner des oppositions. Il est fort possible que l'éducation à l'environnement restera pour longtemps à la marge de l'institution scolaire parce que 
l'alternative qu'elle propose est encore trop radicale. Cela rend d'autant plus nécessaire d'organiser un espace de transaction.

15 Bonne lecture.

\section{BIBLIOGRAPHIE}

Cros, F. (2004). L'innovation scolaire au risque de son évaluation. Paris : L'Harmattan. 